\title{
Injection of Bone Marrow Aspirate for Glenohumeral Joint Osteoarthritis: A Pilot Randomized Control Trial
}

\author{
Tim Dwyer, M.B.B.S., Ph.D., Graeme Hoit, M.D., Adrienne Lee, M.D., Elyse Watkins, \\ Patrick Henry, M.D., Tim Leroux, M.D., Christian Veillette, M.D., \\ John Theodoropoulos, M.D., Darrell Ogilvie-Harris, M.D., and Jaskarndip Chahal, M.D.
}

\begin{abstract}
Purpose: To compare the efficacy of a single, intra-articular, nonconcentrated bone marrow aspirate (BMA) injection in comparison to cortisone for the treatment of glenohumeral joint osteoarthritis (GHJ OA). Methods: Inclusion criteria were patients between the ages of 18 and 75 with a diagnosis of GHJ OA on radiograph. Patients were randomized to receive an ultrasound-guided, intra-articular cortisone injection or BMA injection (without concentration). The primary outcome measure was the Western Ontario Osteoarthritis of the Shoulder (WOOS) index at 12 months. Secondary outcome measures were the QuickDASH, EuroQOL 5-dimensions 5-level questionnaire (EQ-5D-5L) and visual analogue scale. Results: The study included 25 shoulders of 22 patients who completed baseline and 12 months' patient-reported outcome measures ( 12 shoulders received cortisone, 13 shoulders received BMA) after the study was terminated early by changes in Health Canada regulations. Baseline characteristics demonstrated a significant difference in the ages of the 2 groups, with the BMA group being older (61.6 vs 53.8 mean years, $P=0.021$ ). For the BMA group, a significant improvement was seen in the WOOS index $(P=0.002)$, the QuickDASH $(P<0.001)$, and the EQ-5D-5L pain dimension $(P=0.004)$ between baseline and 12 months. No significant difference was seen for any outcome in the cortisone group between baseline and 12 months. No significant difference was demonstrated between changes in the WOOS scores from baseline to 12 months when compared between groups $(P=0.07)$. However, a significant difference in changes in scores was seen in the QuickDASH $(P=0.006)$ and the EQ-5D-5L pain scores $(P=0.003)$ and the EQ-5D-5L health scores $(P=0.032)$ in favor of BMA. Conclusions: The results of this study demonstrate that patients with GHJ OA treated with BMA have superior changes in the QuickDASH and EQ-5D-5L pain and health scores but not in the WOOS outcomes measures at 12 months post injection when compared to patients treated with cortisone. However, because of the limited number of patients as a result of the early termination of the study, larger randomized studies are required to confirm these findings. Level of Evidence: Level II, randomized controlled trial.
\end{abstract}

G lenohumeral joint (GHJ) osteoarthritis (OA) is a painful, progressive and debilitating condition that impairs quality of life. Currently, the most common surgical approach is total shoulder arthroplasty, a major operation that carries with it significant risks, including infection, ${ }^{1-3}$ and in young or active people, a significant rate of early revision surgery. ${ }^{2,4,5}$ Therefore, the goal of early shoulder OA management should be symptom control in order to postpone or prevent the need for joint replacement.

Initial nonoperative management options include physical therapy, pain-control medications and intraarticular injections. Corticosteroid injections are the most commonly used injectables for GHJ OA, although the limited evidence available suggests that pain relief occurs for only short periods post treatment. ${ }^{6}$
From the Women's College Hospital (T.D., E.W., J.T., J.C.), Toronto, Ontario, Canada; Mt Sinai Hospital (T.D., J.T.), Toronto, Ontario, Canada; Sunnybrook Health Sciences Centre (P.H.), Toronto, Ontario, Canada; Toronto Western Hospital (T.L., C.V., D.O.-H.), Toronto, Ontario, Canada; and University of Toronto Orthopaedic Sports Medicine (T.D., G.H., A.L., E.W., P.H., T.L., C.V., J.T., D.O.-H., J.C.), Toronto, Ontario, Canada.

Full ICMJE author disclosure forms are available for this article online, as supplementary material.

Received December 11, 2020; accepted July 13, 2021.
Address correspondence to Tim Dwyer, M.B.B.S., Ph.D.,Women's College Hospital, 76 Grenville Street,Toronto,Ontario,Canada.E-mail: tim.dwyer@ wchospital.ca

(C) 2021 THE AUTHORS. Published by Elsevier Inc. on behalf of the Arthroscopy Association of North America. This is an open access article under the CC BY-NC-ND license (http://creativecommons.org/licenses/by-nc-nd/4.0/). 2666-061X/201979

https://doi.org/10.1016/j.asmr.2021.07.005 
Intra-articular hyaluronic acid injections have been proposed as an alternative to corticosteroid injections, and they have demonstrated a clinically significant improvement in pain and function for as long as 6 months after injection, ${ }^{7,8}$ although with limited effect size. ${ }^{9}$ There is limited information regarding the use of platelet-rich plasma for GHJ OA. ${ }^{10}$

Bone marrow aspirate (BMA) and bone marrow aspirate concentrate (BMAC) injections are relatively recent options for the treatment of OA. Typically taken from the patient's pelvis, BMA has been shown to contain high levels of white blood cells and platelets as well as anti-inflammatory growth factors and cytokines. ${ }^{11}$ BMA also contains mononuclear cells, of which about $0.001 \%$ are mesenchymal stem cells (MSCs), with chondrogenic potential and a paracrine effect in increasing growth factor and cytokine levels. ${ }^{12,13}$ Intraarticular injections of BMA and BMAC in the setting of knee OA have reported statistically significant benefits in improved pain scores and function, ${ }^{14}$ although recent level 1 studies failed to demonstrate its superiority to saline ${ }^{15}$ or to platelet-rich plasma (PRP). ${ }^{16} \mathrm{At}$ this time, there is limited evidence for the use of BMA or BMAC for the treatment of GHJ OA. ${ }^{17,18}$

The purpose of this study was to compare the efficacy of a single, intra-articular, nonconcentrated BMA injection with cortisone for the treatment of GHJ OA. We hypothesized that BMA would improve patientreported outcome measures (PROMs) in comparison to cortisone in the setting of GHJ OA at 12 months post injection.

\section{Methods}

This study was approved by the institutional research ethics board and registered with ClinicalTrials.gov. Inclusion criteria were men or women between the ages of 18 and 75 with a diagnosis of primary GHJ OA on standardized radiographs (anteroposterior, scapula lateral and axillary lateral) who consented to the study between June 2016 and May 2019. Exclusion criteria were patients with a prior condition resulting in secondary OA (such as trauma, dislocation, avascular necrosis, fracture, inflammatory conditions, rotator cuff arthropathy), previous injection of cortisone or other substances, previous surgical interventions, and inability to comply with rehabilitation or form completion, as well as workplace compensation or lawsuit involvement. All BMA kits were supplied by Marrow Cellutions (Ranfac, Avon, MA). No other funding was received from Marrow Cellutions, nor was the company involved in study design, and at no time were they permitted to access the study's data.

Patients presenting with a primary diagnosis of Samilson and Prieto stage 1 (osteophytes $<3 \mathrm{~mm}$ ), stage 2 (osteophytes between 3 and $7 \mathrm{~mm}$ with slight joint irregularity), or stage 3 (osteophytes $>7 \mathrm{~mm}$ with narrowing and sclerosis of the joint) GHJ OA ${ }^{19}$ were eligible for the study. Those who consented to the study were randomized by a study coordinator to receive an ultrasound-guided, intra-articular cortisone injection (Group 1) or a BMA injection (Group 2) by means of a computerized randomization system (sealedenvelope. com) with a 1:1 allocation between the 2 treatment arms. No sham bone marrow aspirations were performed; thus, patients were not blinded to their treatment group.

Patients randomized to Group 1 received an intraarticular injection of $80 \mathrm{mg}$ Depo Medrol with $4 \mathrm{~mL}$ of $1 \%$ lidocaine in the office setting, in line with standard treatment practice at our institution. Patients randomized to Group 2 had BMA taken from the posterior superior iliac spine using the Marrow Cellutions bone marrow aspiration system (Ranfac) in an outpatient procedure room. The posterior superior iliac spine was chosen on the basis of evidence that it contains consistently high concentrations of colony-forming units. $^{20}$ The BMA system was chosen because of familiarity by the surgeons and white-paper evidence of high levels of colony-forming units without the need for a concentration process. Patients were placed in a prone position, and $10 \mathrm{~mL}$ of $0.25 \%$ lidocaine were used for local anesthesia at the donor site. All instrumentation was rinsed with 2,000 units/mL of heparin prior to use, with $.5 \mathrm{~mL}$ of the heparin solution retained in the aspirate syringe. After palpation of landmarks, the introducer needle was inserted past the cortex into the medullary space of the posterosuperior iliac spine, the sharp stylet was removed, and $1 \mathrm{~mL}$ of marrow was aspirated to ensure proper positioning. A blunt stylet was then inserted, and the access needle was advanced $2 \mathrm{~cm}$ farther. Following this, the blunt stylet was removed, and the aspiration cannula and syringe were attached. After each $1 \mathrm{~mL}$ of aspirate, the handle was rotated counterclockwise before repeating the $1 \mathrm{~mL}$ aspirate, as per manufacturer's instructions. This was repeated until $10 \mathrm{~mL}$ of aspirate were obtained. Instrumentation was removed and dressings applied. The BMA was not centrifuged or manipulated in any way.

The injection procedure was identical in both groups. Patients sat upright, with the appropriate shoulder marked and prepped with $2 \%$ chlorhexidine gluconate and $70 \%$ isopropyl alcohol. Under ultrasound guidance, local anesthetic ( $5 \mathrm{~mL}$ of $0.5 \mathrm{~mL}$ lignocaine) was injected down to capsule of the GHJ using a posterior approach. Following this, using an 18-gauge needle under ultrasound guidance, $10 \mathrm{~mL}$ of unmanipulated $\mathrm{BMA}$ or $5 \mathrm{~mL}$ of the local anesthetic/cortisone mix was injected into the glenohumeral joint. Two orthopedic surgeons performed all bone marrow aspirations and ultrasound-guided injections of both cortisone and BMA. 
Subjects in both treatment groups followed the same protocol post procedure. Patients were instructed to rest for 5 days before resuming normal activities and to avoid the use of nonsteroidal anti-inflammatory medication for 2 weeks (although they were allowed to take anti-inflammatories until the day of injection). Physiotherapy was not recommended for either group post injection because not all patients were able to access physiotherapy equally. Patients were followed-up at 3 , 6 and 12 months by a research coordinator blinded to the patients' interventions.

The primary outcome measure was a change in the Western Ontario Osteoarthritis of the Shoulder (WOOS) index from baseline to 12 months postintervention. ${ }^{21}$ The WOOS index is a disease-specific, patient-reported survey composed of 19 questions and providing scores in 4 domains: (1) physical symptoms, (2) sport, recreation and work, (3) lifestyle, and 94) emotions. Each question is answered on a visual analogue scale, with possible scores ranging from 0 to 100 and with total scores ranging from 0 to 1900; a score of 1900 indicates an extreme decrease in shoulder-related quality of life. ${ }^{22}$
The secondary outcome measures used were the QuickDash, EQ-5D-5L and visual analogue scale for pain. The QuickDash ${ }^{23,24}$ is an abbreviated version of the original Disabilities of the Arm, Shoulder and Hand (DASH) outcome measure, ${ }^{25}$ a region-specific PROM composed of 11 items rather than the original 30-item outcome measure. The QuickDASH score has 2 components: the disability/symptom section (11 items, scored 1-5) and the optional sport and work modules (4 items, scored 1-5). Patients use a 5-point Likert scale form, with the final disability/symptom score ranging from 0 (no disability) to $100 \%$ (most severe disability). The minimal clinically important difference for QuickDash is $8 \%$ in individuals with shoulder pain. ${ }^{26}$

The EQ-5D-5L is a general health-status outcome score with 2 parts. The EQ-5D-5L descriptive system comprises 5 dimensions: mobility, self-care, usual activities, pain/ discomfort, and anxiety/depression. ${ }^{27}$ Each dimension has 5 levels. The EQ visual analogue scale records the patient's self-reported health between 0 (the worst health you can imagine) and 100 (the best health you can imagine).
Fig 1. CONSORT (Consolidated Standards of Reporting Trials) flow diagram.

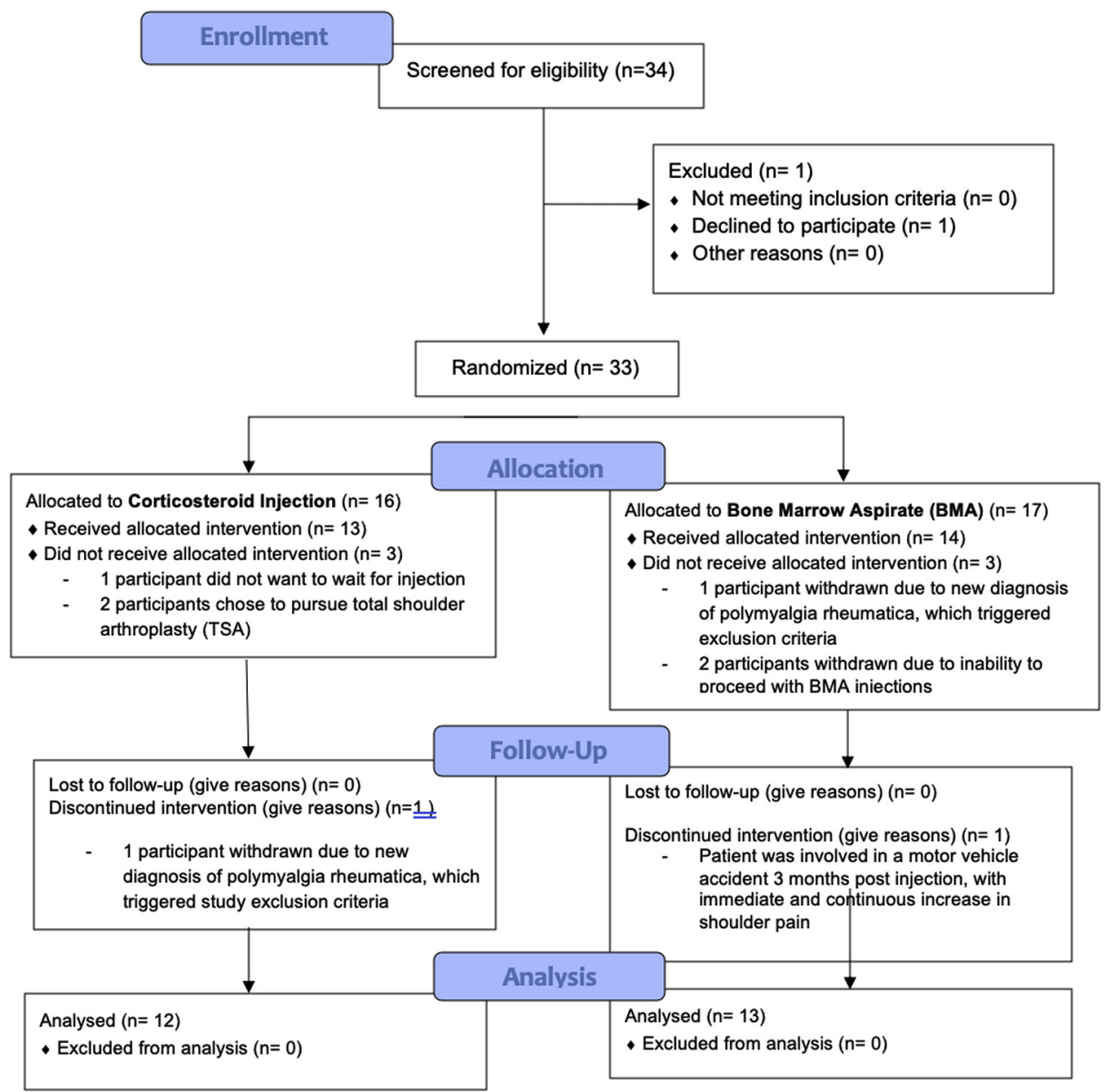


Table 1. Baseline Characteristic Comparison Between BMA and Cortisone Injection Groups

\begin{tabular}{|c|c|c|c|}
\hline & $\begin{array}{c}\text { BMA } \\
\mathrm{N}=13 \\
\end{array}$ & $\begin{array}{c}\text { Cortisone } \\
\mathrm{N}=12\end{array}$ & $P$ Value \\
\hline \multicolumn{4}{|l|}{ Patient demographics } \\
\hline Age, mean (SD) & $61.6(8.1)$ & $53.8(8.3)$ & $0.021^{*}$ \\
\hline \multicolumn{4}{|l|}{ Shoulder characteristics } \\
\hline Affected side, $\mathrm{n}$ right $(\%)$ & $5(35.7 \%)$ & $6(50 \%)$ & $0.69^{\dagger}$ \\
\hline Radiograph arthritis stage & Stage 1: 1 & Stage 1: 0 & $0.23^{\dagger}$ \\
\hline \multicolumn{4}{|l|}{ Baseline outcome measure scores } \\
\hline WOOS, mean (SD) & $1105.7(205.3)$ & $1088.9(262.8)$ & $0.86^{*}$ \\
\hline QuickDASH, mean (SD) & $44.5(12.2)$ & $39.4(11.1)$ & $0.28^{*}$ \\
\hline Work, mean (SD) & $44.3(22.8)$ & $32.0(22.8)$ & $0.26^{*}$ \\
\hline Sport, mean (SD) & $62.5(22.0)$ & $60.9(11.9)$ & $0.86^{*}$ \\
\hline Pain VAS, mean (SD) & $4.5(2.3)$ & $4.8(1.8)$ & $0.72^{*}$ \\
\hline
\end{tabular}

\section{Statistical Analysis}

The planned sample size was 44 patients, or 22 patients per group. The sample size calculation was conducted with the Cohen $d$ effect size of $0.98,80 \%$ power of $80 \%, 2$-sided significance level of 0.05 , and a $15 \%$ attrition rate using $R$ 3.2.0 with the package $p w r$. The effect size was estimated based on statistics (means and standard deviations) reported in a similar study by Morella et al. ${ }^{6}$ However, due to Health Canada regulations, the study was stopped before the planned sample size had been reached.

Demographic data and baseline outcome scores were calculated and presented using means and standard deviations, medians and interquartile ranges, or counts and proportions where appropriate. In our primary analysis, we used an unpaired 2-sample $t$ test to compare differences between our primary outcome (change in WOOS scores from baseline to 12 months) between treatment groups. For secondary outcome measures, unpaired 2-sample $t$ tests or Wilcoxon rank sum tests were employed to investigate differences between groups. All tests were 2 -sided. The significance level was set at $P<0.05$ for all tests. All statistical analysis was performed using SAS s oftware, version 9.4 (SAS Institute, Cary, NC, USA).

\section{Results}

Between June 2016 and May 2019, a total of 33 patients (including 37 shoulders, 4 with bilateral OA) consented to participate in the trial and were randomized. After enrollment, 8 patients were excluded, leaving a total of 25 patients (29 shoulders) in the study. Fig 1 presents the Consolidated Standards of Reporting Trials (CONSORT) flow diagram. On May 15, 2019, Health Canada advised that all cell therapies were to be considered drugs under the Food and Drugs act, requiring authorization by Health Canada to ensure safety and efficacy. For this reason, on May 15, 2019, the study ceased enrolling patients, leaving 2 patients who were randomized to BMA unable to receive their injections. After receiving an injection, 2 patients were excluded from each group, and 1 patient was excluded after a cortisone injection due to a new diagnosis of polymyalgia rheumatica (this patient had also been randomized to receive BMA in her other shoulder, but was excluded prior to this injection). As a result of the new regulations, 25 shoulders ( 22 patients) were included in the study.

All 22 patients ( 25 shoulders) completed baseline and 12-months PROMs. Twelve patients received a cortisone injection, and 13 patients received BMA. Baseline characteristics are presented in Table 1. A significant difference was seen in the ages of the 2 groups the BMA group was older $(P<0.05)$. No complications were seen in either group.

Table 2 presents the difference between the baseline and 12-month outcome scores for the BMA group and for the cortisone group. For the BMA group, a significant difference was seen for the WOOS, the QuickDASH, the QuickDASH work and sports modules, and the EQ-5D-5L pain dimension. No difference was seen for any outcome in the cortisone group.

Table 3 presents a comparison of the 12-month outcome scores for both groups. No significant difference between the BMA group and the cortisone group was seen for the WOOS, the primary outcome measure. 
Table 2. Differences Between Baseline and 12-month Outcome Scores Within BMA and Cortisone Injection Groups

\begin{tabular}{|c|c|c|c|}
\hline & Baseline Score & 12-Month Score & $P$ Value \\
\hline \multicolumn{4}{|l|}{$\overline{\mathrm{BMA}(\mathrm{N}=13)}$} \\
\hline WOOS, mean (SD) & $1082.0(192.8)$ & $684.0(385.3)$ & 0.002 \\
\hline QuickDASH, mean (SD) & $42.5(10.1)$ & $25.5(8.4)$ & $<0.00^{*}$ \\
\hline $\begin{array}{l}\text { QuickDASH Sport, } \\
\text { mean (SD) }\end{array}$ & $61.4(22.7)$ & $39.2(25.3)$ & 0.003 \\
\hline Pain VAS, mean (SD) & $4.3(2.1)$ & $3.0(2.8)$ & $0.13^{*}$ \\
\hline EQ5D-5L Activities, median (IQR) & $2(2-3)$ & $2(2-2)$ & $0.16^{\dagger}$ \\
\hline EQ5D-5L Pain, median (IQR) & $3(3-4)$ & $2(2-3)$ & 0.004 \\
\hline EQ5D-5L Anxiety, median (IQR) & $1(1-2)$ & $1(1-2)$ & $0.25^{\dagger}$ \\
\hline EQ5D-5L Health Score, median (IQR) & $75(70-85)$ & $81(75-85)$ & $0.27^{\dagger}$ \\
\hline \multicolumn{4}{|l|}{ Cortisone $(\mathrm{N}=12)$} \\
\hline WOOS, mean $(\mathrm{SD})$ & $1088.9(262.8)$ & $1002.7(429.2)$ & $0.54^{*}$ \\
\hline Pain VAS, mean (SD) & $4.8(1.8)$ & $5.1(3.2)$ & $0.61^{*}$ \\
\hline EQ5D-5L Mobility, median (IQR) & $1(1-1)$ & $1(1-1)$ & $0.50^{\dagger}$ \\
\hline EQ5D-5L Selfcare, median (IQR) & $2(1.5-2)$ & $2(1.5-3)$ & $1.00^{\dagger}$ \\
\hline EQ5D-5L Activities, median (IQR) & $3(2-3)$ & $3(2-3)$ & $1.00^{\dagger}$ \\
\hline EQ5D-5L Pain, median (IQR) & $3(2-3.5)$ & $3(2.5-3.5)$ & $0.75^{\dagger}$ \\
\hline EQ5D-5L Anxiety, median (IQR) & $1(1-2)$ & $1(1-2.5)$ & $0.25^{\dagger}$ \\
\hline EQ5D-5L Health Score, median (IQR) & $70(70-75)$ & $67.5(55-72.5)$ & $0.13^{\dagger}$ \\
\hline
\end{tabular}

Bold values indicate statistical significance $(P<0.05)$.

IQR, interquartile range; $n$, number of participants; SD, standard deviation; VAS, Visual Analogue Scale; WOOS, Western Ontario Osteoarthritis of the Shoulder index.

${ }^{*} P$ value produced using 2 -sided paired $t$ test.

${ }^{\dagger} P$ value produced using 2 -sided Wilcoxon signed rank test.

A significant difference was seen for the QuickDASH and the EQ-5D-5L pain and health scores.

Table 4 presents a comparison of the changes in outcome scores from baseline to 12 months for the BMA and cortisone injection groups. Again, no significant difference was seen for the WOOS primary outcome measure. A significant difference was seen for the QuickDASH, the QuickDASH sport module, and the EQ5D-5L pain and health scores in favor of the BMA group.

Table 5 and Fig 2 present PROMs at 3,6 and 12 months; some patients did not provide scores at 3 and 6 months. There was no significant difference in PROMs between the 2 groups at the 3 - and 6-month time periods $(P>0.05)$.

A post hoc power calculation based on the WOOS score, which found that the study was powered to a beta of 0.28 (i.e., $72 \%$ of the time, if there was a real difference in WOOS, it would not have been identified). Based on this pilot data, a follow-up study would require 51 patients per group in order to have a beta of 0.8 .

\section{Discussion}

The findings of this study demonstrate that in comparison to cortisone injections, patients receiving BMA injections showed no significant change between baseline and 12 months on the WOOS primary outcome measure but showed significant improvements on the QuickDASH and on the EQ-5D-5L pain and health scores dimensions. Although there was a nonsignificant difference for superior change in WOOS scores in the BMA group compared to the cortisone group, because of early termination, this study was significantly underpowered, and further study is required to confirm this effect.

The theory behind the use of BMA and BMAC in the treatment of OA has focused on the presence of MSCs, although the exact mechanism of action is still unclear. ${ }^{12}$ Both BMA and BMAC have been shown to contain colony-forming units (CFUs) having surface markings consistent with MSCs, ${ }^{11}$ although MSCs have been shown to represent only $0.001 \%$ to $0.02 \%$ of mononuclear cells, even after centrifugation. ${ }^{12}$ Bone marrow is also a rich source of growth factors and cytokines that decrease cell apoptosis and inflammation several times that of PRP, ${ }^{11}$ and it is thought that nucleated cells in BMA have a paracrine effect by delivering various growth factors and cytokines. ${ }^{13}$ BMAC has also been shown to have significantly higher levels of platelets compared to PRP. ${ }^{11}$

The use of both BMA and BMAC has been increasing in orthopedics, whether it be treating cartilage defects in the $\mathrm{knee}^{28}$ or foot and ankle, ${ }^{29}$ the treatment of bone defects and nonunion, ${ }^{30}$ or the treatment of 
Table 3. Comparison of 12-Month Postinjection Outcomes Between BMA and Cortisone groups

\begin{tabular}{|c|c|c|c|}
\hline & BMA N $=13$ & Cortisone $\mathrm{N}=12$ & $P$ Value \\
\hline WOOS, mean (SD) & $684.0(385.3)$ & $1002.7(429.2)$ & $0.06^{*}$ \\
\hline QuickDASH, mean (SD) & $25.5(8.4)$ & $37.1(18.7)$ & $0.01^{*}$ \\
\hline QuickDASH, Work, mean (SD) & $18.1(13.1)$ & $25.0(16.5)$ & $0.36^{*}$ \\
\hline Pain VAS, mean (SD) & $3.0(2.8)$ & $5.1(3.2)$ & $0.09^{*}$ \\
\hline \multicolumn{4}{|l|}{ EQ5D-5L } \\
\hline EQ5D-5L Mobility, median (IQR) & $1(1-1)$ & $1(1-1)$ & $0.90^{\dagger}$ \\
\hline EQ5D-5L Pain, median (IQR) & $2(2-3)$ & $3(2.5-3.5)$ & $\mathbf{0 . 0 2 8} \mathbf{8}^{\dagger}$ \\
\hline EQ5D-5L Anxiety, median (IQR) & $1(1-2)$ & $1(1-2.5)$ & $0.47^{\dagger}$ \\
\hline EQ5D-5L Health Score, median (IQR) & $81(75-85)$ & $67.5(55-72.5)$ & $0.015^{\dagger}$ \\
\hline \multicolumn{4}{|l|}{ Patient Satisfaction } \\
\hline Satisfaction VAS, median (IQR) & $1.3(0.1-3.5)$ & $6.8(4.1-8.6)$ & $0.06^{\dagger}$ \\
\hline
\end{tabular}

degenerative disc disease. ${ }^{31}$ With regard to the treatment of OA, the most common use has been in the setting of knee OA, and some evidence of efficacy has been shown in nonrandomized trials. ${ }^{14}$ However, in 2017, Shapiro compared a BMAC injection with a contralateral saline injection in 25 patients with bilateral knee OA and saw no significant difference at either 6 months ${ }^{15}$ or 12 months post injection. ${ }^{32}$ Publications regarding the use of BMA or BMAC in the treatment of GHJ OA are extremely limited at this time. In 2015, Centeno published work concerning a series of patients undergoing BMAC injections for both rotator cuff tears and GHJ OA; 34 of $115(29.6 \%)$ patients in this study had GHJ OA, and they showed improvement in the outcome scores. ${ }^{17}$ Darrow et al. used both whole bone marrow and BMAC to treat 32 cases of shoulder OA, demonstrating improvements in pain and function at a mean of 6 months of follow-up. ${ }^{18}$ The results of this pilot study demonstrate that patients with GHJ OA who are treated with injections of BMA have improved outcomes at 12 months in the WOOS and QuickDASH outcome measures, as well as in the EQ-5D-5L pain dimension when compared to baseline. This is encouraging and suggests that further, larger scale research regarding the use of BMA and BMAC in this group of difficult-to-treat patients may be rewarding.

Table 4. Comparison of the Change in Outcome Scores From Baseline to 12 Months Postinjection Between BMA and Cortisone Groups

\begin{tabular}{lccc}
\hline & BMA & Cortisone \\
& $\mathrm{N}=12$ & \multicolumn{1}{c}{$P$ Value } \\
\hline WOOS, mean (SD) & $-398.0(364.1)$ & $-86.2(467.8)$ & $-2.3(14.2)$ \\
QuickDASH, mean (SD) & $-17.0(9.9)$ & $-4.5(15.2)$ & $\mathbf{0 . 0 0 6}$ \\
QuickDASH Work, mean (SD) & $-18.8(12.9)$ & $-5.4(17.1)$ & 0.07 \\
QuickDASH Sport, mean (SD) & $-27.5(21.5)$ & $0.6(3.6)$ & $\mathbf{0 . 0 3 9}$ \\
Pain VAS, mean (SD) & $-1.3(2.9)$ & $0.3(0.6)$ & 0.18 \\
EQ5D-5L & & $0.1(1.0)$ & 0.45 \\
EQ5D-5L Mobility, mean (SD) & $-1(0.5)$ & $0.1(0.8)$ & 0.30 \\
EQ5D-5L Selfcare, mean (SD) & $-0.3(0.9)$ & $0.1(0.7)$ & 0.13 \\
EQ5D-5L Activities, mean (SD) & $-0.6(1.3)$ & $0.3(0.5)$ & $\mathbf{0 . 0 0 3}$ \\
EQ5D-5L Pain, mean (SD) & $-0.9(0.8)$ & $-7.3(13.3)$ \\
EQ5D-5L Anxiety, mean (SD) & $-0.3(1.0)$ & 0.10 \\
EQ5D-5L Health Score, mean (SD) & $2.5(7.5)$ & $\mathbf{0 . 0 3 2}$ \\
\hline
\end{tabular}

Change scores $=12$ month scores-baseline scores.

Bold values indicate statistical significance $(P<0.05)$.

IQR, interquartile range; n, number of participants; SD, standard deviation; VAS, visual analogue scale; WOOS, Western Ontario Osteoarthritis of the Shoulder index.

$P$ values produced using 2 -sided unpaired $t$ test. 
Table 5. Outcome Scores Within BMA and Cortisone Injection Groups

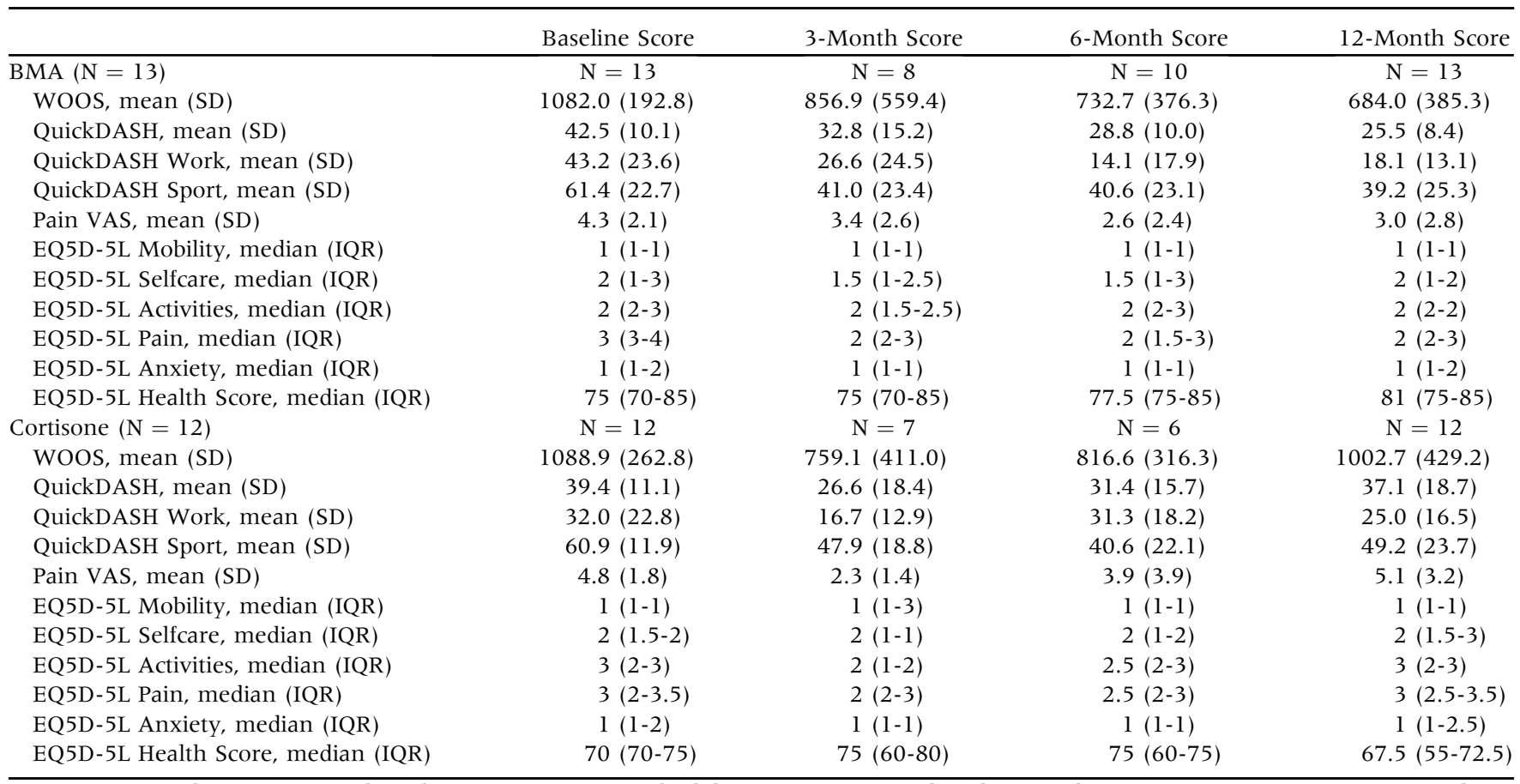

IQR, interquartile range; $n$, number of participants; SD, standard deviation; VAS, visual analogue scale; WOOS, Western Ontario Osteoarthritis of the Shoulder index.

There are a variety of products on the market for BMA and BMAC; 2 recent systematic reviews noted that there was inadequate reporting of both preparation protocols and composition in the literature. ${ }^{33,34}$ In this study, $10 \mathrm{~mL}$ of BMA were extracted from the posterior superior iliac spine using the Marrow Cellutions system and injected in the GHJ without a concentration process. ${ }^{35}$ Alternative systems often use $60 \mathrm{~mL}$ or more of extracted bone marrow, followed by a concentration process to increase the number of MSCs relative to baseline. ${ }^{13}$ However, in both children and adults, it has been shown that after the first few $\mathrm{mL}$ of bone marrow aspiration, any increase in volume results primarily in hemodilution. $^{36,37}$ Further, a study of 30 adults demonstrated that concentrations of MSCs were higher in a $10 \mathrm{~mL}$ sample than in a $50 \mathrm{~mL}$ sample. ${ }^{38}$ The Marrow Cellutions system is closed distally to limit peripheral blood infiltration end of the aspiration cannula and obtains multiple small-volume draws ( $1 \mathrm{~mL}$ ) from side holes during a single puncture. It also uses technology that allows the aspiration needle to be repositioned after each draw. In 1 study, this technique was shown to produce concentrations of CFUs that were comparable to or greater than samples obtained with larger aspiration volumes and concentrations. ${ }^{35}$ However, it is important to note that evidence that improving the numbers of CFUs improves outcomes is limited, ${ }^{39}$ and that further study is required to evaluate the efficacy of different BMA and BMAC systems in the treatment of OA.
There are other, less invasive interventions for the treatment of OA available on the market such as PRP. There is significant evidence for the use of PRP in the setting of knee $\mathrm{OA}$; a systematic review in 2017 found that 9 of 11 randomized controlled trials demonstrated significantly better results with PRP than with hyaluronic acid. ${ }^{40}$ In theory, BMA and BMAC should be more efficacious anti-inflammatory agents than PRP; they have the same or greater levels of platelets as $\mathrm{PRP},{ }^{11}$ provide a source of MSCs not found in peripheral blood, ${ }^{41}$ and are known to contain greater levels of interleukin-1 receptor antagonist, $^{11,42}$ which work to inhibits interleukin-1 catabolism effects. In 2020, Anz et al. published a randomized trial comparing BMAC to PRP in 90 patients with knee OA and demonstrated no significant improvement in pain relief between the 2 groups at 1 year. ${ }^{16}$ At this time there are limited studies looking at the use of PRP in GHJ $\mathrm{OA}$, although studies are currently underway. Because of the superiority of PRP over HA in the setting of knee OA and the potential increased efficacy of BMA and BMAC over PRP, the use of BMA was selected for our study in patients with GHJ OA.

\section{Limitations}

Despite some of the encouraging findings of this study, it is important to acknowledge a risk of underpowered studies such as ours, including type I errors. Furthermore, any findings are not robust because of the small number of cross-over patients required to change a significant finding into a nonsignificant one. ${ }^{43,44}$ For 

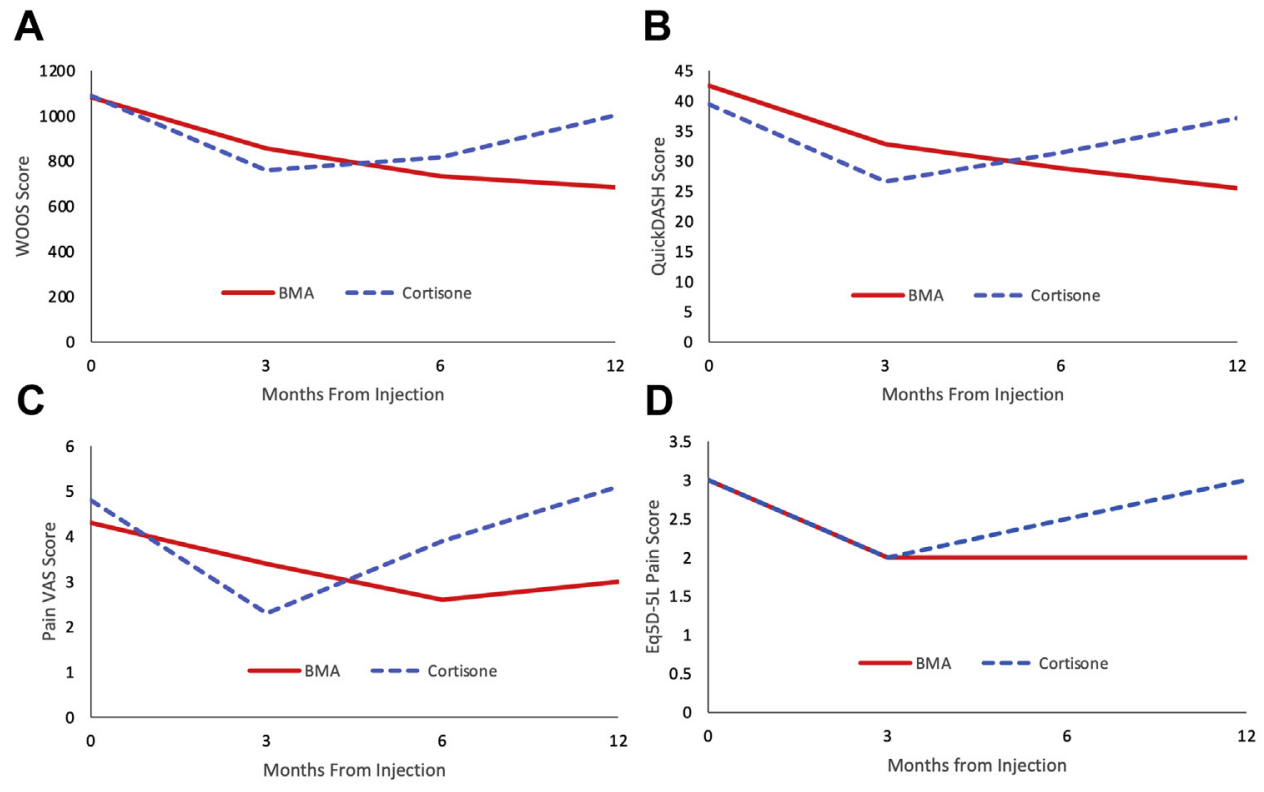

Fig 2. Changes in scores over time for both groups. (A) Western Ontario Osteoarthritis of the Shoulder (WOOS) index. No significant difference was seen between the mean scores for the 2 groups at 12 months post injection $(P=0.06)$. (B) QuickDASH. A significant difference was seen between the mean scores for the 2 groups at 12 months post injection $(P=0.006)$. $(C)$ Pain Visual Analogue Score. No significant difference was seen between the mean scores for the 2 groups at 12 months post injection $(P=0.09)$. (D) Eq5D-5L pain score. A significant difference was seen between the mean scores for the 2 groups at 12 months post injection $(P=0.028)$. A significant difference was also seen for the health score domain $(P=0.015)$, but not for the mobility, selfcare or activities domain. (BMA, bone marrow aspirate.)

example, a recent study looking at significant findings in sports medicine and arthroscopic surgery determined that the median value of the Fragility Index was 2, indicating that changing 2 patients between study groups would reverse the significance. ${ }^{44}$ There were also limited enrollment numbers over the 3 years because some patients preferred a more permanent solution such as arthroplasty.

There are other limitations in this study. The 2 groups were different in age at baseline, although there was no significant difference in baseline outcomes measures between the 2 groups. There were also more patients with severe GHJ OA in the cortisone group. Although randomization usually serves to limit this occurrence, early termination of the study is likely to be the cause. However, this finding may have affected the outcome of our study. Patients were not blinded to the intervention because no sham BMA procedures were performed. For this reason, some of the improvements in the BMA group may be a result of the placebo effect. However, BMA is not without an element of pain and risk to the patient, and the authors of this study did not believe it was ethical to perform sham procedures. Additionally, we did not perform any product sampling, so we cannot comment on the cellular composition of the BMA injections. Furthermore, no follow-up imaging was used because it was believed the primary function of BMA injections was to reduce pain and inflammation as opposed to cartilage regeneration. The follow-up is also relatively short, at only 12 months, and we will endeavor to follow this group for another year. Only patient-reported outcome measures were used in this study because the primary goal of the intervention was to improve pain, not patient strength or range of motion. Likewise, follow-up imaging was not performed because it was not believed that either injection would result in measurable cartilage regeneration. Finally, BMA was used without any concentration process; it may be that BMAC might have resulted an increased therapeutic effect in this group of patients.

\section{Conclusions}

The results of this study demonstrate that patients with GHJ OA that is treated with BMA have superior changes in QuickDASH and EQ-5D-5L pain and health scores but not in WOOS outcomes measures at 12 months post injection, compared to patients treated with cortisone. However, because of the limited number of patients resulting from early termination, larger randomized studies are required to confirm these findings.

\section{References}

1. Anthony CA, Westermann RW, Gao Y, Pugely AJ, Wolf BR, Hettrich CM. What are risk factors for 30-day morbidity and transfusion in total shoulder arthroplasty? 
A review of 1922 cases. Clin Orthop Relat Res 2015;473: 2099-2105.

2. Craig RS, Lane JCE, Carr AJ, Furniss D, Collins GS, Rees JL. Serious adverse events and lifetime risk of reoperation after elective shoulder replacement: Population-based cohort study using hospital episode statistics for England. BMJ 2019;364:1298.

3. Eck CF, Neumann JA, Limpisvasti O, Adams CR. Lack of level I evidence on how to prevent infection after elective shoulder surgery. Knee Surg Sports Traumatol Arthrosc 2018;26:2465-2480.

4. Denard PJ, Raiss P, Sowa B, Walch G. Mid- to long-term follow-up of total shoulder arthroplasty using a keeled glenoid in young adults with primary glenohumeral arthritis. J Shoulder Elbow Surg 2013;22:894-900.

5. Dillon MT, Inacio MC, Burke MF, Navarro RA, Yian EH. Shoulder arthroplasty in patients 59 years of age and younger. J Shoulder Elbow Surg 2013;22:1338-1344.

6. Merolla G, Sperling JW, Paladini P, Porcellini G. Efficacy of Hylan G-F 20 versus 6-methylprednisolone acetate in painful shoulder osteoarthritis: A retrospective controlled trial. Musculoskelet Surg 2011;95:215-224.

7. Kwon YW, Eisenberg G, Zuckerman JD. Sodium hyaluronate for the treatment of chronic shoulder pain associated with glenohumeral osteoarthritis: A multicenter, randomized, double-blind, placebo-controlled trial. J Shoulder Elbow Surg 2013;22:584-594.

8. Zhang B, Thayaparan A, Horner N, Bedi A, Alolabi B, Khan M. Outcomes of hyaluronic acid injections for glenohumeral osteoarthritis: A systematic review and metaanalysis. J Shoulder Elbow Surg 2019;28:596-606.

9. Colen S, Geervliet P, Haverkamp D, Van Den Bekerom MP. Intra-articular infiltration therapy for patients with glenohumeral osteoarthritis: A systematic review of the literature. Int J Shoulder Surg 2014;8:114-121.

10. Rossi LA, Piuzzi NS, Shapiro SA. Glenohumeral osteoarthritis: The role for orthobiologic therapies: Platelet-rich plasma and cell therapies. JBJS Rev 2020;8:e0075.

11. Cassano JM, Kennedy JG, Ross KA, Fraser EJ, Goodale MB, Fortier LA. Bone marrow concentrate and platelet-rich plasma differ in cell distribution and interleukin 1 receptor antagonist protein concentration. Knee Surg Sports Traumatol Arthrosc 2018;26:333-342.

12. Dragoo JL, Guzman RA. Evaluation of the consistency and composition of commercially available bone marrow aspirate concentrate systems. Orthop J Sports Med 2020;8: 2325967119893634.

13. Kim GB, Seo MS, Park WT, Lee GW. Bone marrow aspirate concentrate: Its uses in osteoarthritis. Int J Mol Sci 2020;21:3224-3239.

14. Chahla J, Dean CS, Moatshe G, Pascual-Garrido C, Serra Cruz R, LaPrade RF. Concentrated bone marrow aspirate for the treatment of chondral injuries and osteoarthritis of the knee: A systematic review of outcomes. Orthop J Sports Med 2016:4:2325967115625481.

15. Shapiro SA, Kazmerchak SE, Heckman MG, Zubair AC, O'Connor MI. A prospective, single-blind, placebocontrolled trial of bone marrow aspirate concentrate for knee osteoarthritis. Am J Sports Med 2017;45:82-90.

16. Anz AW, Hubbard R, Rendos NK, Everts PA, Andrews JR, Hackel JG. Bone marrow aspirate concentrate is equivalent to platelet-rich plasma for the treatment of knee osteoarthritis at 1 year: A prospective, randomized trial. Orthop J Sports Med 2020;8:23259671 19900958.

17. Centeno CJ, Al-Sayegh H, Bashir J, Goodyear S, Freeman MD. A prospective multi-site registry study of a specific protocol of autologous bone marrow concentrate for the treatment of shoulder rotator cuff tears and osteoarthritis. J Pain Res 2015;8:269-276.

18. Darrow M, Shaw B, Schmidt N, Boeger G, Budgett S. Treatment of shoulder osteoarthritis and rotator cuff tears with bone marrow concentrate and whole bone marrow injections. Cogent Med 2019;6:1628883.

19. Samilson RL, Prieto V. Dislocation arthropathy of the shoulder. J Bone Joint Surg Am 1983;65:456-460.

20. Otto A, Muench LN, Kia C, et al. Proximal humerus and ilium are reliable sources of bone marrow aspirates for biologic augmentation during arthroscopic surgery. Arthroscopy 2020;36:2403-2411.

21. Lo IK, Griffin S, Kirkley A. The development of a diseasespecific quality of life measurement tool for osteoarthritis of the shoulder: The Western Ontario Osteoarthritis of the Shoulder (WOOS) index. Osteoarthrit Cartil 2001;9: 771-778.

22. Rasmussen JV, Jakobsen J, Olsen BS, Brorson S. Translation and validation of the Western Ontario Osteoarthritis of the Shoulder (WOOS) index: The Danish version. Patient Relat Outcome Meas 2013;4:49-54.

23. Gummesson C, Ward MM, Atroshi I. The shortened Disabilities of the Arm, Shoulder and Hand questionnaire (QuickDASH): Validity and reliability based on responses within the full-length DASH. BMC Musculoskelet Disord 2006;7:44.

24. Matheson LN, Melhorn JM, Mayer TG, Theodore BR, Gatchel RJ. Reliability of a visual analog version of the QuickDASH. J Bone Joint Surg Am 2006;88:1782-1787.

25. Beaton DE, Katz JN, Fossel AH, Wright JG, Tarasuk V, Bombardier C. Measuring the whole or the parts? Validity, reliability, and responsiveness of the Disabilities of the Arm, Shoulder and Hand outcome measure in different regions of the upper extremity. $J$ Hand Ther 2001;14:128-146.

26. Mintken PE, Glynn P, Cleland JA. Psychometric properties of the shortened disabilities of the Arm, Shoulder, and Hand Questionnaire (QuickDASH) and Numeric Pain Rating Scale in patients with shoulder pain. J Shoulder Elbow Surg 2009;18:920-926.

27. Herdman M, Gudex C, Lloyd A, et al. Development and preliminary testing of the new five-level version of EQ-5D (EQ-5D-5L). Qual Life Res 2011;20:1727-1736.

28. Cavinatto L, Hinckel BB, Tomlinson RE, Gupta S, Farr J, Bartolozzi AR. The role of bone marrow aspirate concentrate for the treatment of focal chondral lesions of the knee: A systematic review and critical analysis of animal and clinical studies. Arthroscopy 2019;35:1860-1877.

29. Murphy EP, McGoldrick NP, Curtin M, Kearns SR. A prospective evaluation of bone marrow aspirate concentrate and microfracture in the treatment of osteochondral lesions of the talus. Foot Ankle Surg 2019;25: 441-448.

30. Imam MA, Holton J, Ernstbrunner L, et al. A systematic review of the clinical applications and complications of 
bone marrow aspirate concentrate in management of bone defects and nonunions. Int Orthop 2017;41: 2213-2220.

31. Hirase T, Jack RA 2nd, Sochacki KR, Harris JD, Weiner BK. Systematic review: Is intradiscal injection of bone marrow concentrate for lumbar disc degeneration effective? Cureus 2020;12:e9045.

32. Shapiro SA, Arthurs JR, Heckman MG, et al. Quantitative T2 MRI mapping and 12-month follow-up in a randomized, blinded, placebo controlled trial of bone marrow aspiration and concentration for osteoarthritis of the knees. Cartilage 2019;10:432-443.

33. Murray IR, Robinson PG, West CC, et al. Reporting standards in clinical studies evaluating bone marrow aspirate concentrate: A systematic review. Arthroscopy 2018;34:1366-1375.

34. Piuzzi NS, Hussain ZB, Chahla J, et al. Variability in the preparation, reporting, and use of bone marrow aspirate concentrate in musculoskeletal disorders: A systematic review of the clinical orthopaedic literature. J Bone Joint Surg Am 2018;100:517-525.

35. Scarpone M, Kuebler D, Chambers A, et al. Isolation of clinically relevant concentrations of bone marrow mesenchymal stem cells without centrifugation. J Transl Med 2019;17:10.

36. Yandow SM, Van de Velde SK, Siebert J, Perkins SL. The influence of aspiration volume on the number of osteoblastic progenitors obtained from bone marrow in children. J Pediatr Orthop 2019;39:382-386.

37. Muschler GF, Boehm C, Easley K. Aspiration to obtain osteoblast progenitor cells from human bone marrow: The influence of aspiration volume. J Bone Joint Surg Am 1997;79:1699-1709.

38. Hernigou P, Homma Y, Flouzat Lachaniette $\mathrm{CH}$, et al. Benefits of small volume and small syringe for bone marrow aspirations of mesenchymal stem cells. Int Orthop 2013;37:2279-2287.

39. Pettine KA, Suzuki RK, Sand TT, Murphy MB. Autologous bone marrow concentrate intradiscal injection for the treatment of degenerative disc disease with three-year follow-up. Int Orthop 2017;41:2097-2103.

40. Chevrier A, Darras V, Picard G, et al. Injectable chitosanplatelet-rich plasma implants to promote tissue regeneration: In vitro properties, in vivo residence, degradation, cell recruitment and vascularization. J Tissue Eng Regen Med 2018;12:217-228.

41. Kuznetsov SA, Mankani MH, Leet AI, Ziran N, Gronthos S, Robey PG. Circulating connective tissue precursors: extreme rarity in humans and chondrogenic potential in guinea pigs. Stem Cells 2007;25:1830-1839.

42. Ziegler CG, Van Sloun R, Gonzalez S, et al. Characterization of growth factors, cytokines, and chemokines in bone marrow concentrate and platelet-rich plasma: A prospective analysis. Am J Sports Med 2019;47:2174-2187.

43. Walsh M, Srinathan SK, McAuley DF, et al. The statistical significance of randomized controlled trial results is frequently fragile: A case for a fragility index. J Clin Epidemiol 2014;67:622-628.

44. Khan M, Evaniew N, Gichuru M, et al. The fragility of statistically significant findings from randomized trials in sports surgery: A systematic survey. Am J Sports Med 2017:45:2164-2170. 